\title{
Do molecular tests really differentiate malignant IPMNS from benign?
}

Authors

Institutions
Omer Basar $^{1,2}$, William R. Brugge ${ }^{1}$

${ }^{1}$ Pancreas Biliary Center, Gastrointestinal Unit, Massachusetts General Hospital, Boston, Massachusetts, United States

${ }^{2}$ Department of Gastroenterology, Hacettepe Medical School, Ankara, Turkey submitted 19. October 2016 accepted after revision 25. October 2016

\section{Bibliography}

Dol http://dx.doi.org/

10.1055/s-0042-121004

Endoscopy International Open

2016; 04: E1236-E1237

(c) Georg Thieme Verlag KG

Stuttgart · New York

E-ISSN 2196-9736

\section{Corresponding author}

Omer Basar, MD

3-H Gl Associates,

Zero Emerson Place,

Blossom St. Massachusetts

General Hospital

Boston, MA, 02114

Fax: +1-617-724-5997

obasar@mgh.harvard.edu

\section{License terms}

(ब)(1) $\ominus \circledast$
Bournet et al. have questioned the role of endoscopic ultrasound-guided fine needle aspiration (EUS-FNA) plus KRAS and GNAS mutations in malignant intraductal papillary mucinous neoplasms (IPMNs) of the pancreas in this issue of Endoscopy International Open [1]. Bournet et al. claimed that testing for KRAS mutations in cystic fluid improved the accuracy of results for cytopathologic diagnosis of malignancy whereas GNAS mutation testing did not improve the results. How should clinicians interpret these outcomes and do these results help to detect and treat an IPMN before it progresses to a pancreatic adenocarcinoma?

IPMNs are the most frequently detected type of mucin-producing neoplasm and the exact rate of progression to malignancy has not yet been defined clearly (ranging from 38 to $68 \%$ for Main Duct-IPMNs [MD-IPMNs] and 12 to $47 \%$ for Branch Duct-IPMNs [BD-IPMNs] in surgical series of symptomatic patients) [2]. The goal of any diagnostic test for a pancreatic cystic neoplasm is accurate detection of its malignant potential. Recent guidelines on pancreatic cysts recommend a multimodal diagnostic approach including cross-sectional imaging, EUS-FNA and cyst fluid analysis (such as biochemistry, cytology and molecular analysis) to overcome this complex assessment. Although cross-sectional imaging provides detailed images of the high-risk lesions, use of EUS-FNA has increased the accuracy of diagnosis of advanced neoplasia. Cytology is highly specific but approximately $50 \%$ sensitive for diagnosis of a malignancy arising from IPMN, due to inadequate cellularity in most cases. On the other hand, elevated cyst fluid carcinoembryonic antigen (CEA) level is considered the most accurate test to distinguish a mucinous cyst from non-mucinous. However, CEA alone can be used neither to differentiate IPMN from a mucinous cystic neoplasm nor a malignant IPMN from a noninvasive IPMN.
Several molecular techniques have been designed for further evaluation of pancreatic cystic neoplasms; however, DNA-based assays on aspirated cyst fluid have emerged as the most useful and reproducible tool. Recent studies on DNA sequencing have not only shown the genetic alterations specific for pancreatic cystic neoplasms, but also may help diagnose and differentiate these neoplasms. The most commonly found genetic alteration in IPMNs is KRAS mutation (found in over $80 \%$ of cases). It occurs predominantly in codon 12 , but it may also occur in codons 13 and 61 . KRAS mutations are associated with BD-IPMNs and more often present in pancreatobiliary and gastric type IPMNs. Moreover, GNAS mutation is a unique mutation for IPMNs with a frequency of $58 \%$ to $65 \%$, occurring in codon 201 or 227 . GNAS mutation is mostly found in MD-IPMNs rather than BD-IPMNs and mainly present in intestinal subtype. A mutation in KRAS and/or GNAS is found in over $90 \%$ of IPMNs.

Bournet et al. enrolled 37 IPMN patients with clinical and/or imaging predictors in a 4-year study [1]. The final diagnosis of IPMNs ( $n=10$ were benign and $n=27$ were malignant) was obtained from pancreatic resections $(n=18)$, biopsies during laparotomy, EUS-FNA analysis and follow-ups $(n=19)$. Aspirated cyst fluid was evaluated for cytology. KRAS (codon 12) and GNAS (codon 201) mutation assays were performed using the TaqMan ${ }^{\circledR}$ allelic discrimination on EUS-FNA fluid. KRAS and GNAS assays were successful in all but one sample. The sensitivity, specificity, positive predictive value (PPV) and negative predictive value (NPV) and accuracy of cytology alone to diagnose malignancy in IPMN were 55\%, 100\%, 100\%, $45 \%$ and $66 \%$, respectively. When KRAS mutation analysis was combined with cytology, these values were $92 \%, 50 \%, 83 \%, 71 \%$ and $81 \%$, respectively. GNAS analysis improved performance of neither cytology alone, nor cytology combined with KRAS. The authors concluded that using the 
$\operatorname{TaqMan}^{\circledR}$ allelic discrimination assay was feasible in IPMN-associated malignancy evaluation. Further, although performing GNAS mutation did not add value to diagnosis of malignant IPMNs, the combination of a KRAS mutation with cytology increased the performance of cytology alone for sensitivity, specificity, NPV, accuracy and predicting malignancy to $80 \%$.

The first study evaluating the role of KRAS mutations for malignancy assessment in preoperative pancreatic cyst fluid reported that KRAS mutations were present in 10 of 11 malignant cysts and the sensitivity and specificity of KRAS mutations followed by allelic loss to predict a malignant cyst were $91 \%$ and $93 \%$, respectively [3]. Later a multicenter prospective study (the PANDA study) included 113 patients and reported a higher number of DNA mutations in malignant cysts. However, presence of a KRAS mutation was similar between malignant and premalignant cysts. In the PANDA study, the high-amplitude KRAS mutation followed by allelic loss had $96 \%$ specificity but $37 \%$ sensitivity for malignancy [4]. Another study by the same group revealed long-term follow-up results in 63 patients and found that presence of KRAS mutation was associated with progression of malignancy [5]. In a study including 618 patients, KRAS mutation was found to be $54 \%$ sensitive and $100 \%$ specific for a mucinous cyst and combining KRAS mutation with an elevated cyst fluid CEA level increased sensitivity to $83 \%$ with specificity unchanged at $85 \%$ [6]. On the other hand, CEA alone was found to be $63 \%$ sensitive and $88 \%$ specific for differentiation of a mucinous cyst from non-mucinous in a meta-analysis of 12 studies [7].

In our investigation of pancreatic cysts, we examined 943 patients with KRAS results and found $48 \%$ sensitivity, $100 \%$ specificity, $100 \%$ positive predictive value, and $47 \%$ negative predictive value (NPV) for KRAS mutation in a mucinous cyst. In the same cohort of patients, sensitivity improved to $75 \%$ and NPV to $60 \%$ when a KRAS mutation was combined with CEA elevation. Moreover, 56 patients in this cohort had a malignant cyst (34 adenocarcinoma and 22 high-grade dysplasia [HGD]) and KRAS mutation was more frequent in malignant mucinous cysts than in benign tumors (73.2\% - 37.3\%). The NPVs of KRAS mutation alone and together with CEA elevation for a malignant cyst were $77.6 \%$ and $83.3 \%$, respectively. In our study, we suggested that although the diagnostic value of KRAS mutation positivity for malignant cysts remains limited, because it lacks specificity for malignant and non-malignant differentiation, the high NPV might help to exclude a malignant cyst in clinical practice [8].

With the addition of GNAS mutation tests, the sensitivity of molecular analysis for detection of a mucinous cyst has increased. Detection of KRAS and/or GNAS mutation had 65\% sensitivity and $100 \%$ specificity for mucinous cyst detection in a study [9]. Interestingly, although almost all of the studies suggest that a GNAS mutation is unique for IPMNs, a recent study found GNAS mutation in 2 patients with serous cystadenoma [10] and we found one in a patient with pseudocyst [11]. A recent study included 38 patients with resected malignant IPMNs that had sufficient tissue for micro-dissection and showed that KRAS and GNAS mutations did not differ according to the degree of neoplasia (KRAS: invasive IPMN 71\%, HGD 62\%, low-grade dysplasia 74\%; GNAS: invasive IPMN 61\%, HGD 59\%, low-grade dysplasia 53\%)
[12]. A recent meta-analysis of 36 studies revealed that KRAS and GNAS mutations could be diagnostic markers for IPMN, however, neither KRAS nor GNAS mutations were associated with the malignant potential or prognosis in patients with IPMN [13].

DNA-based assays have improved in recent years. When compared with conventional Sanger sequencing, next-generation sequencing is highly specific and sensitive for detection of pancreatic cysts that have malignant potential. Besides, the other advantages of next-generation sequencing are that it requires smaller amounts of DNA for analysis and it can simultaneously assay multiple genes. Although KRAS and/or GNAS mutation tests alone may help diagnose IPMNs, it is difficult to say if these mutations can replace classification and prognostication with multimodal diagnostic methods. Further studies (combination with TP53, PTEN and PICK3CA) are needed to establish the concordance of these tests with diagnosis and prognosis of pancreatic cystic neoplasms.

\section{Competing interests: None}

\section{References}

1 Bournet B, Vignolle-Vidoni A, Grand D et al. Endoscopic ultrasoundguided fine-needle aspiration plus KRAS and GNAS mutation in malignant intraductal papillary mucinous neoplasm of the pancreas. Endosc Int Open 2016; 04: 1228-1235

2 Stark A, Donahue TR, Reber HA et al. Pancreatic Cyst Disease: A Review. JAMA 2016; 315: 1882 - 1893

3 Khalid A, McGrath KM, Zahid M. The role of pancreatic cyst fluid molecular analysis in predicting cyst pathology. Clin Gastroenterol Hepatol 2005; 3: 967 - 973

4 Khalid A, Zahid M, Finkelstein SD. Pancreatic cyst fluid DNA analysis in evaluating pancreatic cysts: a report of the PANDA study. Gastrointest Endosc 2009; 69: 1095-1102

5 Rockacy MJ, Zahid M, McGrath KM. Association between KRAS mutation, detected in pancreatic cyst fluid, and long-term outcomes of patients. Clin Gastroenterol Hepatol 2013; 11: 425 - 429

6 Nikiforova MN, Khalid A, Fasanella KE. Integration of KRAS testing in the diagnosis of pancreatic cystic lesions: a clinical experience of 618 pancreatic cysts. Mod Pathol 2013; 26: 1478 - 1487

7 Thornton GD, McPhail MJ, Nayagam S. Endoscopic ultrasound guided fine needle aspiration for the diagnosis of pancreatic cystic neoplasms: a meta-analysis. Pancreatology 2013; 13: 48 - 57

8 Kadayifci A, Al-Haddad M, Atar M. The value of KRAS mutation testing with CEA for the diagnosis of pancreatic mucinous cysts. Endosc Int Open 2016; 4: E391 - 396

9 Singhi $A D$, Zeh HJ, Brand RE. American Gastroenterological Association guidelines are inaccurate in detecting pancreatic cysts with advanced neoplasia: a clinicopathologic study of 225 patients with supporting molecular data. Gastrointest Endosc 2016; 8: 1107-1117

10 Lee LS, Doyle LA, Houghton J. Differential expression of GNAS and KRAS mutations in pancreatic cysts. JOP 2014; 15: 581 - 586

11 Kadayifci A, Atar M, Wang JL. Value of adding GNAS testing to pancreatic cyst fluid KRAS and carcinoembryonic antigen analysis for the diagnosis of intraductal papillary mucinous neoplasms. Dig Endosc 2016 Aug 11 DOI 10.1111/den.12710

12 Tan MC, Basturk O, Brannon AR. GNAS and KRAS Mutations Define Separate Progression Pathways in Intraductal Papillary Mucinous Neoplasm-Associated Carcinoma. J Am Coll Surg 2015; 220: 845-854

13 Lee JH, Kim Y, Choi JW et al. KRAS, GNAS, and RNF43 mutations in intraductalpapillary mucinous neoplasm of the pancreas: a meta-analysis. Springerplus 2016; 5: 1172 